\title{
Human papillomavirus is a favourable prognostic factor in cancer of unknown primary in the head and neck region and in hypopharyngeal cancer (Review)
}

\author{
LARS SIVARS $^{1}$, CINZIA BERSANI $^{1}$, NATHALIE GRÜN ${ }^{1}$, TORBJÖRN RAMQVIST ${ }^{1}$, \\ EVA MUNCK-WIKLAND ${ }^{2}$, CHRISTIAN VON BUCHWALD ${ }^{3}$ and TINA DALIANIS ${ }^{1}$ \\ ${ }^{1}$ Department of Oncology-Pathology; ${ }^{2}$ Department of Clinical, Technical Sciences and Intervention, \\ Karolinska Institutet, Stockholm, Sweden; ${ }^{3}$ Department of Otolaryngology, Head and Neck Surgery \\ and Audiology, Rigshospitalet, Copenhagen University Hospital, Copenhagen, Denmark
}

Received April 26, 2016; Accepted September 8, 2016

DOI: $10.3892 / \mathrm{mco} .2016 .1050$

\begin{abstract}
Human papillomavirus (HPV), in addition to smoking and alcohol, is a cause of oropharyngeal squamous cell carcinoma (OPSCC), particularly of the tonsils and base of the tongue (TSCC and BOTSCC, respectively). Moreover, HPV-positive TSCC and BOTSCC are associated with a better outcome compared with their HPV-negative counterparts (80 vs. 40\% 3-year disease-free survival rate, respectively) and their incidence has increased in several countries. Recently, accumulating evidence of HPV in a considerable proportion of cancers of unknown primary (CUP) in the head and neck region and in a small proportion of hypopharyngeal SCCs has been reported. Furthermore, HPV-positive tumours, particularly cases with HPV DNA positivity in combination with overexpression of p16, also tend to have a better clinical outcome compared with that of the corresponding HPV-negative tumours. This finding is particularly prominent in HPV-positive CUPs of the head and neck region, where the primary tumour likely originates from the oropharynx. Thus, the determination of HPV status and p16 expression may be of value for the diagnosis and treatment of CUP of the head and neck region and may also be of value for hypopharyngeal cancers in the future. However, for hypopharyngeal cancer as well as other non-OPSCCs, additional studies per subsite on the effect of HPV status on survival are required.
\end{abstract}

Correspondence to: Dr Tina Dalianis, Department of Oncology-Pathology, Karolinska Institutet, SE-171 76 Stockholm, Sweden

E-mail: tina.dalianis@ki.se

Key words: human papillomavirus, cancer of unknown primary, hypopharyngeal cancer, head and neck cancer, oropharyngeal cancer

\section{Contents}

1. Introduction

2. CUP of the head and neck region

3. HPV in hypopharyngeal cancer

4. HPV in non-OPSCC sites other than CUP and hypopharyngeal cancer

5. Conclusions

\section{Introduction}

In 2007, human papillomavirus (HPV) type 16 was defined, in addition to smoking and alcohol, as a risk factor for oropharyngeal squamous cell carcinoma (OPSCC) by the International Agency for Research on Cancer (1). Among OPSCC sites, HPV is mostly found in SCCs of the tonsils and the base of tongue (TSCC and BOTSCC, respectively), which differ from the corresponding HPV-negative tumours (1). Most notably, patients with HPV-positive TSCC and BOTSCC have a significantly better prognosis compared with those with the HPV-negative counterparts and other head and neck SCCs (HNSCCs), with a 5-year disease-free survival (DFS) rate of 80 vs. $40 \%$, respectively (1-4). Furthermore, in several Western countries, the incidence of TSCC and BOTSCC has increased, mainly due to an increase in the incidence of HPV infection; however, the fraction of HPV-positive tumours varies between countries, which is likely due to different cultural and smoking habits $(1,5-7)$.

In countries where smoking has declined, HPV-positive TSCC and BOTSCC account for a larger proportion of HNSCC, a transition affecting treatment strategies (1). Lately, HNSCC therapy (including HPV-positive OPSCC) has been intensified, with hyperfractionated radiotherapy (RT), chemotherapy and targeted therapy added to the previously administered conventional RT or extensive surgery, causing more serious side effects (1). Intensive therapy is not required for all HPV-positive TSCCs and BOTSCCs, and efforts have been focused on identifying additional prognostic markers in combination with HPV, in order to select patients for 
de-escalated RT and chemotherapy, or for minimal invasive surgery, such as robotic surgery $(1,8,9)$.

In addition, attention has been directed towards identifying HPV at other head and neck sites. Accumulating reports describe the presence of HPV DNA in several cancers of unknown primary (CUP) in the head and neck region, as well as in a minority of hypopharyngeal cancers and other non-OPSCC locations (10-27). Notably, patients with HPV-positive tumours, particularly in CUP of the head and neck region and possibly also in hypopharyngeal cancer, tend to have a better clinical outcome compared with those with corresponding HPV-negative tumours, in whom HPV status may also have implications for diagnosis and therapy (10-25,27,28). However, this has not been investigated in the same detail for other cancer locations in the head and neck region, whereas reports usually compare survival between patients with HPV-positive and those with HPV-negative non-OPSCC.

The aim of the present study was to investigate certain aspects of the effect of HPV on clinical outcome for CUP of the head and neck region and hypopharyngeal cancer.

\section{CUP of the head and neck region}

The first sign of head and neck cancer is often a lump in the neck, i.e., a lymph node metastasis. The diagnostic work-up with fine-needle aspiration cytology (FNAC), computed tomography (CT) including the chest, and primary tumour biopsies if a tumour is evident, eventually reveal the primary site. In $1.5-9 \%$ of the cases, however, a primary tumour may not be identified, despite a systematic clinical work-up including tonsillectomy, random biopsies from the base of the tongue and nasopharynx, and magnetic resonance imaging of the head and neck (17). In such cases, the lymph node metastasis is defined as CUP of the head and neck region (10-17). FNAC showing a cancer other than SCC, e.g. adenocarcinoma, is indicative of a primary tumour likely situated outside the head and neck region, while a SCC suggests a HNSCC (10). It was previously suggested that CUPs at different sites of the body exhibit similar biological behaviour, with early progression and metastasis. However, CUP (SCC) of the head and neck region often resembles advanced HNSCC and has a 35-50\% 5-year DFS, while CUP at other sites has a median survival significantly lower than 1 year (10-17). To confirm the cytological diagnosis with histopathology and as part of the treatment, the preferred approach in several centres remains neck dissection and removal of lymph nodes from levels I-II to IV-V (depending on the centre). An SCC metastasis in level II or III of the neck likely originates from an OPSCC, while a metastasis further down may suggest a different origin.

Recent studies reported the prevalence of HPV DNA, p16 or both in CUP of the head and neck region (10-17). The highest HPV prevalence (59-92\%) was observed in patients who were not subjected to tonsillectomy or random biopsies from the base of the tongue, who were at a higher risk of missing a minute TSCC or BOTSCC (17). In patients who had undergone tonsillectomy and random biopsies, the HPV prevalence was lower (28-52\%) (17). Furthermore, the application of robotic surgery to the diagnostic work-up of patients classified as CUP, comprising the removal of lymphoid tissue localised superficially in the base of the tongue, has showed promising results and improvement of the detection rate of the primary tumours (8). Thus, a variation in HPV prevalence in CUP of the head and neck region is to be expected, depending on the diagnostic procedure; furthermore, HPV prevalence differs widely for OPSCC between countries and over time (1,5-7).

It has been demonstrated that HPV-positive CUP of the head and neck region has a significantly better prognosis compared with that of HPV-negative CUP (10-17). In one report, $40 \%$ of CUP cases were HPV-positive and had a 3 -year overall survival (OS) rate of $80 \%$, compared with $36.7 \%$ in the HPV-negative cases, with similar DFS (14). In another study, $22 \%$ of the cases were HPV-positive and had a clear OS benefit, also when adjusted for advanced age in a multivariate Cox regression analysis (15). The fact that the survival of HPV-positive CUP was similar to that of HPV-positive TSCC and BOTSCC, further supports the hypothesis that HPV-positive CUP may originate from these sites (13-15). Other studies have shown a trend towards better survival, but did not reach statistical significance, possibly due to the limited size of the samples $(11,12)$.

\section{HPV in hypopharyngeal cancer}

The presence of HPV has also been investigated in hypopharyngeal cancer with varying results. In our previous study, the prevalence of HPV in hypopharyngeal cancer in Stockholm, Sweden, was investigated in cases diagnosed between 2000 and 2007 (20). The presence of HPV DNA was detected in $7 / 109(6 \%)$ of the tumours that were available for evaluation, and 4/7 HPV-positive tumours were HPV16-positive (3.7\%) and also overexpressed p16, with $3 / 4$ of the latter patients surviving for $>5$ years (20). There was, however, no statistically significant difference in survival between patients with HPV-positive/p16-overexpressing tumours compared with patients with HPV-negative hypopharyngeal cancer, which is likely due to the limited number of HPV16 DNA-positive/p16-overexpressing cases (20). The remaining 3 HPV-positive tumours did not overexpress p16 (20). In the same study, p16 overexpression was observed in $14 \%$ of the hypopharyngeal cancer cases, and was not found to be a suitable surrogate marker for the presence of HPV DNA; furthermore, it was not correlated with survival (20). This study was later followed up to determine whether the proportion of HPV-positive tumours had increased in the Stockholm region during the years 2008-2013, and whether the combined presence of HPV16 DNA and p16 overexpression was correlated with survival (22). The prevalence of HPV16 had not increased and was 3.7\% (22). Notably, among the 142 patients diagnosed in 2000-2011 with hypopharyngeal cancer in Stockholm, Sweden, the ones with tumours that were HPV16 DNA-positive and overexpressed p16, had a significantly better 3 -year OS $(\mathrm{P}=0.0185)$ compared with others (22). In addition, a similar tendency was observed for disease-specific survival (DSS) $(\mathrm{P}=0.0581)$. Furthermore, the 3-year OS and DSS were $86 \%$ for patients with HPV16 DNA-positive/p16-overexpressing tumours, which was very similar to that reported for patients with HPV DNA/p16-positive TSCC/BOTSCC $(1,3,4)$. Thus, the limited prevalence of HPV16 DNA combined with p16 overexpression indicates that HPV16 is not a major cause of hypopharyngeal cancer, but it may confer a prognostic benefit (22). 
Other studies reported an HPV prevalence of $0-82 \%$, with a recent meta-analysis showing an average of $21.9 \%$ (18-27). The majority of the studies in the meta-analysis differed from the study by Ernoux-Neufcoeur et al, which reported an $82 \%$ HPV prevalence, as only $9 \%$ of the tumours overexpressed p16, suggesting that, in that study, only a limited number of tumours truly had an active HPV infection $(19,21)$.

High-risk HPV infection as a prognostic positive marker was also demonstrated in a study by Joo et al (24). In this report, high-risk HPV DNA determined by in situ hybridization was present in 7/64 (10.9\%) of hypopharyngeal SCC cases, and was more commonly found in cancers of the pyriform sinus compared with other hypopharyngeal cancer sites. Furthermore, patients with HPV-positive hypopharyngeal cancer had a better DFS $(\mathrm{P}=0.026)$ and a better DSS $(\mathrm{P}=0.047)$ compared with those with HPV-negative cancer (24).

Finally, in a cohort similar to ours $(20,22)$, but significantly smaller (27 patients), it was not possible to correlate p16 overexpression with survival or the presence of HPV DNA (25).

\section{HPV in non-OPSCC sites other than CUP and hypopharyngeal cancer}

HPV has also been demonstrated at other non-OPSCC subsites in the head and neck region, although its prevalence is generally significantly lower compared with that in $\operatorname{OPSCC}(26,27)$. Furthermore, fewer studies report the effect of HPV per subsite, and when grouped together, the impact of HPV on survival is generally not significant (27). However, certain studies indicate that the presence of HPV DNA together with p16 overexpression, or the presence of p16 overexpression alone, should be taken into account in patients with laryngeal cancer, while others suggest the opposite $(28,29)$.

\section{Conclusions}

Accumulating evidence demonstrates that HPV16 DNA, also most often accompanied by p16 overexpression, is frequently found in CUP of the head and neck, and patients with this profile have a better prognosis compared with those with HPV-negative CUP, similar to patients with HPV-positive TSCC and BOTSCC. SCC cytology and positive HPV16 DNA and p16 status in CUP may therefore allow for treatment directed towards the base of the tongue and palatine tonsils, including radiotherapy/chemoradiotherapy or a minimally invasive surgical approach alone, comprising transoral robotic surgery with corresponding neck dissection.

In hypopharyngeal SCC, HPV16 DNA was not as frequently found as in CUP of the head and neck region, and overexpression of p16 cannot be used as a surrogate marker for HPV or as a predictive marker for survival. However, patients with HPV16 DNA-positive p16-overexpressing hypopharyngeal cancer tend to have a better clinical outcome compared with those with HPV-negative cancer. For hypopharyngeal cancer, where the choice between primary surgery and primary radiotherapy/chemoradiotherapy is a major issue, HPV status together with overexpression of p16 may prove to be of use for decision-making. However, further studies on the effect of HPV status on survival, in hypopharyngeal cancer as well as in other individual non-OPSCC subsites, would be justified.

\section{Acknowledgements}

The present study was supported in part by grants from the Swedish Cancer Foundation, the Stockholm Cancer Society, the Cancer and Allergy Foundation, the Stockholm City Council and the Karolinska Institutet, Sweden.

\section{References}

1. Dalianis T: Human papillomavirus and oropharyngeal cancer, the epidemics, and significance of additional clinical biomarkers for prediction of response to therapy (Review). Int J Oncol 44: 1799-1805, 2014

2. Marklund L, Näsman A, Ramqvist T, Dalianis T, Munck-Wikland E and Hammarstedt L: Prevalence of human papillomavirus and survival in oropharyngeal cancer other than tonsil or base of tongue cancer. Cancer Med 1: 82-88, 2012.

3. Lindquist D, Romanitan M, Hammarstedt L, Näsman A, Dahlstrand H, Lindholm J, Onelöv L, Ramqvist T, Ye W, Munck-Wikland E and Dalianis T: Human papillomavirus is a favourable prognostic factor in tonsillar cancer and its oncogenic role is supported by the expression of E6 and E7. Mol Oncol 1: 350-335, 2007.

4. Attner P, Du J, Näsman A, Hammarstedt L, Ramqvist T, Lindholm J, Marklund L, Dalianis T and Munck-Wikland E: Human papillomavirus and survival in patients with base of tongue cancer. Int J Cancer 128: 2892-2897, 2011.

5. Sturgis EM and Cinciripini PM: Trends in head and neck cancer incidence in relation to smoking prevalence: An emerging epidemic of human papillomavirus-associated cancers? Cancer 110: 1429-1435, 2007.

6. Näsman A, Attner P, Hammarstedt L, Du J, Eriksson M, Giraud G, Ahrlund-Richter S, Marklund L, Romanitan M, Lindquist D, et al: Incidence of human papillomavirus (HPV) positive tonsillar carcinoma in Stockholm, Sweden: An epidemic of viral-induced carcinoma? Int J Cancer 125: 362-366, 2009.

7. Chaturvedi AK, Engels EA, Pfeiffer RM, Hernandez BY, Xiao W, Kim E, Jiang B, Goodman MT, Sibug-Saber M, Cozen W, et al: Human papillomavirus and rising oropharyngeal cancer incidence in the United States. J Clin Oncol 29: 4294-4301, 2011.

8. Channir HI, Rubek N, Nielsen HU, Kiss K, Charabi BW, Lajer CB and von Buchwald C: Transoral robotic surgery for the management of head and neck squamous cell carcinoma of unknown primary. Acta Otolaryngol 135: 1051-1057, 2015.

9. Tertipis N, Hammar U, Näsman A, Vlastos A, Nordfors C, Grün N, Ährlund-Richter A, Sivars L, Haeggblom L, Marklund L, et al: A model for predicting clinical outcome in patients with human papillomavirus-positive tonsillar and base of tongue cancer. Eur J Cancer 51: 1580-1587, 2015.

10. Begum S, Gillison ML, Nicol TL and Westra WH: Detection of human papillomavirus-16 in fine-needle aspirates to determine tumor origin in patients with metastatic squamous cell carcinoma of the head and neck. Clin Cancer Res 13: 1186-1191, 2007.

11. Compton AM, Moore-Medlin T, Herman-Ferdinandez L, Clark C, Caldito GC, Wang XI, Thomas J, Abreo FW and Nathan CO: Human papillomavirus in metastatic lymph nodes from unknown primary head and neck squamous cell carcinoma. Otolaryngol Head Neck Surg 145: 51-57, 2011.

12. Tribius S, Hoffmann AS, Bastrop S, Görögh T, Haag J, Röcken C, Clauditz T, Grob T, Wilczak W, Tennstedt P, et al: HPV status in patients with head and neck of carcinoma of unknown primary site: HPV, tobacco smoking, and outcome. Oral Oncol 48: 1178-1184, 2012.

13. Park GC, Lee M, Roh JL, Yu MS, Choi SH, Nam SY, Kim SY and Cho KJ: Human papillomavirus and p16 detection in cervical lymph node metastases from an unknown primary tumor. Oral Oncol 48: 1250-1256, 2012.

14. Sivars L, Näsman A, Tertipis N, Vlastos A, Ramqvist T, Dalianis T, Munck-Wikland E and Nordemar S: Human papillomavirus and p53 expression in cancer of unknown primary in the head and neck region in relation to clinical outcome. Cancer Med 3: 376-384, 2014. 
15. Jensen DH, Hedback N, Specht L, Høgdall E, Andersen E, Therkildsen MH, Friis-Hansen L, Norrild B and von Buchwald C: Human papillomavirus in head and neck squamous cell carcinoma of unknown primary is a common event and a strong predictor of survival. PLoS One 9: e110456. 2014.

16. Davis KS, Byrd JK, Mehta V, Chiosea SI, Kim S, Ferris RL, Johnson JT and Duvvuri U: Occult primary head and neck squamous cell carcinoma: Utility of discovering primary lesions. Otolaryngol Head Neck Surg 151: 272-278, 2014.

17. Boscolo-Rizzo P, Schroeder L, Romeo S and Pawlita M: The prevalence of human papillomavirus in squamous cell carcinoma of unknown primary site metastatic to neck lymph nodes: A systematic review. Clin Exp Metastasis 32: 835-845, 2015.

18. Hoffmann M, Görögh T, Gottschlich S, Lohrey C, Rittgen W, Ambrosch P, Schwarz E and Kahn T: Human papillomaviruses in head and neck cancer: 8 year-survival-analysis of 73 patients. Cancer Lett 218: 199-206, 2005.

19. Ernoux-Neufcoeur P, Arafa M, Decaestecker C, Duray A, Remmelink M, Leroy X, Herfs M, Somja J, Depuydt CE, Delvenne P and Saussez S: Combined analysis of HPV DNA, p16, p21 and p53 to predict prognosis in patients with stage IV hypopharyngeal carcinoma. J Cancer Res Clin Oncol 137: 173-181, 2011.

20. Wendt M, Romanitan M, Näsman A, Dalianis T, Hammarstedt L, Marklund L, Ramqvist T and Munck-Wikland E: Presence of human papillomaviruses and p16 expression in hypopharyngeal cancer. Head Neck 36: 107-112, 2014.

21. Ndiaye C, Mena M, Alemany L, Arbyn M, Castellsagué X, Laporte L, Bosch FX, de Sanjosé S and Trottier H: HPV DNA, E6/E7 mRNA, and p16INK4a detection in head and neck cancers: A systematic review and meta-analysis. Lancet Oncol 15: 1319-1331, 2014.

22. Dalianis T, Grün N, Koch J, Vlastos A, Tertipis N, Nordfors C, Näsman A, Wendt M, Romanitan M, Bersani C, et al: Human papillomavirus DNA and p16(INK4a) expression in hypopharyngeal cancer and in relation to clinical outcome, in Stockholm, Sweden. Oral Oncol 51: 857-861, 2015.
23. Rodrigo JP, Hermsen MA, Fresno MF, Brakenhoff RH, García-Velasco F, Snijders PJ, Heideman DA and García-Pedrero JM: Prevalence of human papillomavirus in laryngeal and hypopharyngeal squamous cell carcinomas in northern Spain. Cancer Epidemiol 39: 37-41, 2015.

24. Joo YH, Lee YS, Cho KJ, Park JO, Nam IC, Kim CS, Kim SY and Kim MS: Characteristics and prognostic implications of high-risk HPV-associated hypopharyngeal cancers. PLoS One 8: e78718.2013.

25. Wilson DD, Rahimi AS, Saylor DK, Stelow EB, Jameson MJ, Shonka DC, Reibel JF, Levine PA and Read PW: p16 not a prognostic marker for hypopharyngeal squamous cell carcinoma. Arch Otolaryngol Head Neck Surg 138: 556-561, 2012.

26. Castellsagué X, Alemany L, Quer M, Halec G, Quirós B, Tous S, Clavero O, Alòs L, Biegner T, Szafarowski T, et al: HPV Involvement in head and neck cancers: Comprehensive assessment of biomarkers in 3680 patients. J Natl Cancer Inst 108: djv403, 2016.

27. Chung CH, Zhang Q, Kong CS, Harris J, Fertig EJ, Harari PM, Wang D, Redmond KP, Shenouda G, Trotti A, et al: p16 protein expression and human papillomavirus status as prognostic biomarkers of nonoropharyngeal head and neck squamous cell carcinoma. J Clini Oncol 32: 3930-3938. 2014.

28. Shaughnessy JN, Farghaly H, Wilson L, Redman R, Potts K, Bumpous J, Silverman C and Dunlap NE: HPV: A factor in organ preservation for locally advanced larynx and hypopharynx cancer? Am J Otolaryngol 35: 19-24. 2014.

29. Young RJ, Urban D, Angel C, Corry J, Lyons B, Vallance N, Keid S, Iseli TA, Solomon B and Rischin D: Frequency and prognostic significance of p16(INK4A) protein overexpression and transcriptionally active human papillomavirus infection in laryngeal squamous cell carcinoma. Br J Cancer 112: 1098-1104. 2015. 